\title{
I BENI CULTURALI TRA RICERCA TUTELA VALORIZZAZIONE E GESTIONE
}

\author{
DANIELE MANACORDA (*)
}

SUNTO. - Il patrimonio culturale dell'Italia è così largamente diffuso che non può essere gestito solo dallo Stato. Occorre guardare al patrimonio con gli occhi dei cittadini, senza cedere di un pollice sul ruolo delle competenze tecniche e professionali. Ricerca, tutela, valorizzazione e gestione del patrimonio culturale sono cose ben diverse, che però si danno la mano: perché la ricerca ci fa capire il senso delle cose; la tutela ci dice come proteggerle; la valorizzazione ci dice come conservarne il senso diffondendone la percezione; la gestione ci dice come continuare a poterlo fare.

$* * *$

ABSTRACT. - The cultural heritage of Italy is so widespread that it can not be managed by the State alone. We must look at the heritage with the eyes of citizens, without giving up a single inch on the role of technical and professional skills. Research, protection, enhancement and management are four very different things, that go, however, hand in hand: because the research helps us understand the meaning of things; the protection tells us how to safeguard them; enhancement tells us how to preserve the sense of the heritage disseminating its social perception; the management tells us how to keep doing it.

Credo di non esagerare se apro questa mia relazione osservando che il dibattito in corso da due anni sul patrimonio culturale valga almeno venti degli anni trascorsi, se pensiamo all'apatia che accompagnò, 12 anni fa, la nascita del Codice Urbani a conclusione di un periodo che aveva pur visto, con i decreti Veltroni di fine millennio, qualche barlume di innovazione almeno sul fronte della valorizzazione dei beni culturali.

(*) Università Roma Tre, Centro Linceo Interdisciplinare 'B. Segre', Roma, Italia. E-mail: daniele.manacorda@uniroma3.it 
La temperie attuale è certo merito del dinamismo di un ministro che - a volte senza guardare in faccia a nessuno - ha mosso le acque dello stagno; e merito di una pubblicistica che ha visto impegnati alcuni addetti ai lavori ${ }^{1}$, scatenando reazioni più o meno composte, che ci hanno almeno fatto prendere atto che la stagione del pensiero unico, e del timore manifestato da tanti media nel toccare temi apparentemente di esclusivo dominio di una casta, è finita. Anche se stiamo in mezzo al guado: condizione scomoda, ma vitale e, foss'anche solo per questo, liberatoria.

La riforma dei poli museali e quella che ha istituito le soprintendenze uniche ${ }^{2}$ sono state due spallate, che hanno demolito da un lato d'un colpo - per dirla con Roberto Cecchi ${ }^{3}$ - il moloch che per decenni ha bloccato ogni iniziativa considerando inseparabile il ruolo della tutela dalla fruizione; e dall'altro la falsa coscienza di una tutela contestuale affermata negli intenti e negata nell'organizzazione amministrativa.

Queste due riforme hanno preso le mosse dall'assunto - quasi rivoluzionario - che a un certo punto deve pur arrivare il momento in cui non si può più continuare a fare quello che si è sempre fatto. E questo semplicemente perché - come più volte ha sottolineato Massimo Montella - il patrimonio culturale non rappresenta un valore in sé, dato a priori, quanto piuttosto un valore che in tanto è capace di operare in quanto sia percepito come tale, cioè come un valore culturale, da una larga parte della popolazione.

1 Bibliografia essenziale della più recente saggistica sul tema: M. Montella, Il capitale culturale, Macerata 2009; A. Carandini, Il nuovo dell'Italia è nel passato, RomaBari 2012; S. Settis, Azione popolare. Cittadini per il bene comune, Torino 2012; G.M. Golinelli (a cura di), Patrimonio culturale e creazione di valore, Padova 2012; B. Zanardi, Un patrimonio artistico senza. Ragioni, problemi, soluzioni, Milano 2013; T. Montanari, Istruzioni per l'uso del futuro. Il patrimonio culturale e la democrazia che verrà, Roma 2014; D. Manacorda, L'Italia agli italiani. Istruzioni e ostruzioni per il patrimonio culturale, Bari 2014; C. Tosco, I beni culturali. Storia, tutela e valorizzazione, Bologna 2014; T. Montanari, Privati del patrimonio, Torino 2015; G. Volpe, Patrimonio al futuro, Milano 2015; R. Cecchi, Abecedario. Come proteggere e valorizzare il patrimonio culturale italiano, Milano 2015. Si aggiunga ora anche L. Casini, Ereditare il futuro. Dilemmi sul patrimonio culturale, Bologna 2016.

2 DPCM, 29 agosto 2014, n. 171; DM, 23 gennaio 2016, n. 44.

3 Cecchi, Abecedario, 83. 
Non voglio peccare di ottimismo, ma un'aria nuova dunque circola perché sembrano essersi mossi all'unisono alcuni pezzi delle istituzioni e alcuni settori dell'opinione pubblica, almeno quelli stufi di rimanere schiacciati tra le sciatterie del 'tira a campare' e certe prediche dei nostalgici del Grand Tour. Perplessità, incomprensioni, debolezze di tutti gli attori in campo non mancano e non mancheranno. E occorre saper ascoltare le voci discordi, distinguendo quelle dettate dalla paura del nuovo da quelle desiderose di contribuire comunque ad una innovazione, che non è mai buona in sé, ma la cui qualità si misura sempre con l'esistente.

Assistiamo spesso a prese di posizioni che allargano il solco piuttosto che ricucirlo. Il che può essere un bene, se impedisce pasticci concettuali e false unanimità, ma paralizzante se si cristallizza in due sfere non comunicanti.

Lasciatemi anche dire che - sarà forse un inguaribile modo di vedere le cose da archeologo - l'approccio alla filiera ricerca/tutela/ valorizzazione/gestione del patrimonio culturale passa anche da una presa di coscienza storica, che ci invita a mettere a fuoco i problemi del presente alla luce di quel che è successo nei cento anni che ci separano dalle prime leggi di tutela dello Stato unitario. Ma un approccio storico ha bisogno anche della consapevolezza che viviamo in un mondo che corre con la velocità di una cometa, nei rapporti di forza internazionali, nell'incontro/scontro fra culture, nel fluttuare delle ricchezze dall'una all'altra parte del pianeta, nell'impero della comunicazione globale che ci fa vivere la vita degli altri in tempo reale. Sono tutti fenomeni che si riflettono potentemente in quel meccanismo che regola il flusso delle informazioni e anche il turismo di massa globalizzato, da cui ci sentiamo come soffocati e del quale non possiamo e non vogliamo fare a meno, mentre vorremmo ancora provare a godere del nostro incomparabile patrimonio anche semplicemente come cittadini.

Lo stesso concetto di patrimonio evoca una dimensione valoriale, che investe qualità che vanno ben al di là della percezione estetica e monumentale di derivazione idealistica ${ }^{4}$. E questo sin dalla defini-

4 Di grande impatto in tal senso la lettura di M. Montella (a cura di), Economia e gestione dell'eredità culturale. Dizionario metodico essenziale, Padova 2016, e in particolare della Introduzione al volume da parte di E. Borgonovi e M. Montella (XXI-XXX). 
zione di bene culturale proposta mezzo secolo fa dalla Commissione Franceschini ${ }^{5}$ e ampliata ed alimentata dalla maturazione epistemologica che ha investito le discipline di riferimento, in primis l'archeologia contemporanea, che opera secondo premesse storiche ed antropologiche mille miglia lontane dai primordi antiquari e storico-artistici, di cui vuol tuttavia conservare i lasciti più vitali'

Sì che mai come oggi abbiamo la consapevolezza che le politiche pubbliche debbano orientare l'azione di tutela su un patrimonio diffuso, ponendo quindi al centro dell'attenzione la qualità del territorio, ed investendo su di essa con alcuni corollari che possono di volta in volta spaziare nel campo della conservazione, avviando finalmente progetti di manutenzione preventiva programmata strategici per la salvaguardia del patrimonio e per l'occupazione, che ribaltino la prassi consolidata di costosi restauri che, non conoscendo il bene, intervengono episodicamente quando il danno è fatto; o possono riguardare la messa in campo di forme di "valorizzazione condivisa in cui lo stato conservi il ruolo di indirizzo e controllo coinvolgendo la parte privata nella progettualità e nella valorizzazione" .

Insomma, direi quasi che il nostro sistema di tutela ha funzionato fino a quando il suo orizzonte è rimasto limitato a un numero circoscritto di beni e fino a quando gli utenti del sistema sono stati prevalentemente ristretti ad una fascia sociale che era già educata alla loro comprensione, sì che l'assioma tutela=valorizzazione poteva sembrare difendibile. Con una massa sempre più imponente di beni e di aree, da considerare vincolati senza saper bene perché, di cui si è faticato a cogliere la natura relazionale, il sistema - come dice Cecchi - ha iniziato a comparire opaco, burocratico, capriccioso, discrezionale ${ }^{8}$. E l'amministrazione ha preso a sbandare perché i compiti sono andati via via sovrastando la struttura organizzativa. Il sistema non ha seguito l'evolversi della nozione di patrimonio culturale e non è mai stato organizza-

5 F. Franceschini (a cura di), Per la salvezza dei beni culturali in Italia. Atti e documenti della Commissione d'indagine per la tutela e la valorizzazione del patrimonio storico, archeologico, artistico e del paesaggio, I-III, Roma 1967.

6 Mi permetto di rinviare alla voce Archeologia in Montella (a cura di), Economia e gestione, 24-29, con relativa bibliografia.

7 Cecchi, Abecedario, 107.

8 Cecchi, Abecedario, 111. 
to per la valorizzazione?. In poche parole, abbiamo assistito anche qui ad un mancato aggiornamento delle categorie interpretative della realtà di fronte al salto di scala planetario della contemporaneità.

Ben pochi degli strumenti messi a punto sinora sul cammino che collega ricerca, tutela, valorizzazione e gestione ci sembrano ancora validi oggi ${ }^{10}$. Sono mutate le categorie esterne a noi e al nostro mondo specialistico; è cambiato il senso e la pratica delle discipline, che si tratti della storia dell'arte, del diritto, dell'architettura che vuole progettare sull'antico, della comunicazione che vuole diffondere il senso del patrimonio culturale, e fa da megafono a un pubblico potenziale di miliardi di voci, magari così diverse da noi, ma non per questo prive di una loro coerenza.

Non posso soffermarmi su questo versante, ma occorre riflettere anche sul fatto che - come ci ricorda Giulio Volpe ${ }^{11}$ - la comunicazione non è una concessione paternalistica, che presuppone un atteggiamento fondamentalmente pedagogico: è il motore stesso di tutela e valorizzazione. Ciò significa che semplicità e chiarezza comunicativa sono innanzitutto una scelta etica, il prodotto di un impegno civile degli addetti ai lavori, che non coincide con l'impegno scientifico. E per questo purtroppo un certo umanesimo elitario finisce col presentarsi come il rovescio della medaglia del turismo barbarico. La mancanza in tutto il territorio nazionale di musei delle città e dell'Italia, che sappiano raccontare non singoli capolavori, ma ampi contesti geografici e storici, presentando i nostri monumenti in modo comprensibile, è una delle ragioni del "mordi e fuggi" 12 .

Per questo occorre studiare un po' anche il nostro recente passato per capire noi addetti ai lavori chi siamo e da dove veniamo, con quali categorie in testa pensiamo di fare la nostra parte davanti a problemi a volte più grandi di noi, o dovrei dire più grandi di chiunque, ma davanti ai quali non possiamo sottrarci dal produrre idee e proposte, sapendo che i monumenti e i siti storici non sono certo né di noi archeologi che

9 Cecchi, Abecedario, 136-146.

10 P. Petraroia, La valorizzazione come dimensione relazionale della tutela, in G. Negri-Clementi e S. Stabile (a cura di), Il diritto nell'arte. 3. La protezione del patrimonio artistico, Milano 2015, 41-49.

11 Volpe, Patrimonio, 71.

12 Si veda D. Manacorda, Musei della città: qualche osservazione di carattere generale sul caso di Roma, Città e storia, 3 (2008), 225-236. 
li scaviamo né degli architetti che li trasformano, né dei professori che ne scrivono libri, né dei funzionari che ne rallentano il degrado.

Ma senza di noi questo patrimonio starebbe meglio o peggio? Vi sembrerà una domanda stupida: ma certo che starebbe peggio, anzi chissà quanto ne sarebbe stato distrutto! Ma quale conservazione proponiamo noi di questo patrimonio? A quali esigenze pensiamo di dover dare una risposta? Con quali strumenti pensiamo di trovarla? Non è che l'amore del patrimonio qualche volta è troppo geloso? o miope? o santo? o contento di sé? Tante cose sono cambiate in questo secolo abbondante. Ma non la difficoltà che noi addetti ai lavori, e di conseguenza la politica, ancora proviamo nel misurarci con la finalità sociale delle nostre competenze specialistiche. Invece di indugiare in lamentele catastrofistiche e insieme autoassolutorie, che ci bombardano quotidianamente, dobbiamo invece imparare a riflettere di più sulle nostre responsabilità di interfacce pensanti tra un patrimonio sconfinato e una platea umana globalizzata, nelle cui mani è il suo destino.

Il rapporto tutela/valorizzazione è uno dei nodi del contendere. Ricerca, tutela, valorizzazione e gestione appartengono certo alla stessa filiera, sono tra loro intimamente incastrate, ma evitiamo di raccontarci che sarebbero la stessa cosa. È questa insulsa storiella che ci ha fatto nascondere la testa nella sabbia, annegati in un pensiero confermativo, che cerca conforto nei mantra invece che nell'etica del fare. Veramente ha ragione Montella ${ }^{13}$ quando osserva che, per come se ne tratta sui mezzi di informazione, la valorizzazione dei beni culturali sembra consistere in una disputa surreale fra chi vuole e chi rifiuta eventi e spettacoli all'interno di monumenti e musei. È una cosa ridicola, che lascio a chi, di fatto insultando la stessa Soprintendenza che lo progetta, già pontifica che il restauro dell'arena del Colosseo sarà kitsch e degno di Las Vegas ${ }^{14}$. Quella vicenda, che mi ha coinvolto direttamente ${ }^{15}$, è un esempio paradigmatico del paradosso di una cultura che vuole salvare un patrimonio e al tempo stesso non si accorge di affossarlo. È un episodio illuminante, dove benaltrismo, feticismo, iperstoricismo e cata-

13 M. Montella, Beni culturali. La valorizzazione che serve, La Stampa, 17 aprile 2015, 23.

14 R. Merlo, Pioggia di soldi sul Colosseo per un futuro stile Las Vegas, La Repubblica, 5 agosto 2015.

15 D. Manacorda, Anfiteatri e campi di golf, Archeo, 343 (2014), 94-96. 
strofismo sono andati a braccetto nell'assoluta indifferenza al dato reale della conservazione del bene e della plateale assenza di servizi.

Valorizzare vuol dire promuovere e soddisfare al meglio la richiesta di usare i luoghi del patrimonio culturale e di comprenderne il valore. Si tratta semplicemente di questo. Che poi tanto semplice non è, perché questo comporta che si sappia comunicare il loro senso storico ed estetico, ragionando su linguaggio e strumenti, e anche, scusate se è poco, che oggetti e luoghi siano tenuti in buone condizioni.

È quindi ovvio che la frequentazione di musei, monumenti e parchi vada resa quanto più piacevole, curando l'aspetto dei luoghi, l'accoglienza, la professionalità del personale, i servizi offerti, che non sono mai aggiuntivi ma costitutivi. Si può certamente passare un'ora in un museo $\mathrm{o}$ in un sito a contemplare le opere, fermando il tempo, ma anche mezza giornata in letizia con familiari o amici, e aver voglia di ritornarci. E mi piace ricordare qui a Milano il pensiero concreto in tal senso di Renato Bazzoni ${ }^{16}$.

Detto ciò, entrano in campo promozione e marketing; e la produzione di eventi e spettacoli, che magari richiamano anche chi altrimenti non scoprirebbe mai quel monumento o quel museo. Gli spettacoli, di qualunque genere, possono dare benefici economici diretti e indiretti, ma servono soprattutto a integrare i monumenti nel nostro vivere quotidiano, anziché confinarli nel turismo. Servono a far crescere il numero di cittadini interessati a ritornarci e quindi a vederli tutelati. Servono ad allargare la base sociale degli alleati del patrimonio.

Se poi la valorizzazione investe il 'museo diffuso' sul territorio italiano, non sarà solo il valore e il senso dei singoli monumenti che occorrerà spiegare, ma le relazioni e l'insieme che le accoglie, con la cultura materiale e immateriale, le tradizioni e i riti, i saperi e i saper fare, le feste e i gusti, compresi quelli di prodotti tipicamente italiani, che possiedono anche loro un valore culturale costruito nei millenni. Se hanno anche un valore monetario e occupazionale, questo è un ulteriore motivo per sostenerne la tutela, che dunque non è l'antitesi della valorizzazione, ma ne è semmai la premessa e la conseguenza, in un circolo virtuoso che dà senso al valore riconosciuto dalla ricerca ${ }^{17}$. Perché la ricer-

16 R. Bazzoni, Tutta questa bellezza, a cura di A. Cicalò Danioni, Milano 2014.

17 Cfr. M. Montella, Che cosa significa 'valorizzare' un bene culturale, La Stampa, 12 agosto 2015, 25. 
ca ci fa capire il senso delle cose; la tutela ci dice come proteggerle; la valorizzazione ci dice come conservarne il senso diffondendone la percezione; la gestione ci dice come continuare a poterlo fare ${ }^{18}$.

Quindi ricerca/tutela/valorizzazione/gestione sono quattro cose diverse, che si danno la mano: il loro concatenamento dovrebbe essere al centro anche della formazione universitaria, come purtroppo non $\mathrm{e}^{19}$. Per chiudere sul rapporto tra tutela e valorizzazione, lasciatemi esprimere un pensiero paradossale, che è questo: che cosa distingue in fondo queste due funzioni? Forse il fatto che la prima è sostenuta prioritariamente da una solida erudizione, nella migliore accezione del termine, che permette di classificare il bene nel tempo e nello spazio e quindi di riconoscerlo; la seconda si esplica invece come vero e proprio fatto culturale, che - uscendo dal perimetro specialistico - ne riverbera il senso nel contesto sociale più ampio, avviando un processo di moltiplicazione del suo valore, producendo quindi cultura. Altro che mercificazione: la valorizzazione è il senso stesso di ogni attività di conoscenza socialmente intesa.

Un uomo dello Stato come Roberto Cecchi ha affermato di recente che occorre "azzerare le attività gestionali" dello Stato nel campo del patrimonio culturale, perché "l'amministrazione non ha mai fatto della gestione un progetto, trattandola come un'attività di risulta, come se fosse un compito che spetta ad altri" ${ }^{20}$; ma - aggiungo io - tenendosela ben stretta. "Quel che lo Stato deve fare - afferma Cecchi ${ }^{21}$ - è altro. Deve favorire rapporti di cooperazione fra portatori di interesse e cioè tra il sistema istituzionale e quello economico territoriale, non solo per cercare sponsorizzazioni, ma soprattutto per stimolare lo sviluppo dell'offerta culturale e creativa". Per questo occorre respingere quelle posizioni impaurite, che gridano alla fine della funzione pubblica in favore di una paventata iniziativa privata. Il conflitto non è tra pubblico e privato, ma tra interesse pubblico e interesse privato ${ }^{22}$.

18 Si veda il mio intervento su Patrimonio culturale: un diritto collettivo, in $\mathrm{La}$ democrazia della conoscenza. Patrimoni culturali, sistemi informativi e open data: accesso libero ai beni comuni?, Atti del Convegno (Trieste 28-29 gennaio 2016), a cura di R. Auriemma, Udine 2017, 117-123.

19 Sul tema, tuttavia centrale, della formazione non posso soffermarmi in questa sede: si veda in proposito Volpe, Patrimonio, 44-51.

20 Cecchi, Abecedario, 132.

21 Cecchi, Abecedario, 107.

22 Volpe, Patrimonio, 60. 
Un patrimonio così largamente diffuso non può gestirlo solo lo Stato. La rivoluzione copernicana di cui abbiamo bisogno è quella che ci aiuta a guardare al patrimonio con gli occhi dei cittadini, dei visitatori, degli utenti (non solo con quelli del cardinal Pacca o del maestro di buona volontà), senza cedere di un solo millimetro (ma è un millimetro importante) sul ruolo delle competenze tecnico-scientifiche e professionali. Se l'ipertrofia delle specializzazioni crea sempre più tecnici di altissimo livello, che faticano a contestualizzare il senso e il fine della propria opera, l'obiettivo ambizioso è quello di ricomporre l'unità dei saperi nella concretezza dei progetti, senza cedere in nulla sulla qualità degli interventi sul piano sia teorico che operativo. Perché è da questa qualità che dipende il raggiungimento del fine primo del nostro operare, ovvero "l'incremento del capitale intellettuale delle persone e, conseguentemente, della qualità di vita delle collettività. Ma per questo obiettivo non si può far leva sul valore posizionale dell'offerta, su una comunicazione che per contenuti e per linguaggio esclude tutti quelli che non coltivano interessi disciplinari specialistici e, dunque, la maggioranza delle persone"23.

Ragionare sul senso della valorizzazione significa anche ragionare sui casi positivi che ne documentano la praticabilità e gli effetti: piccoli esempi dal basso o grandi esempi organizzativi, sostenuti da un pensiero forte, come è l'esperienza del FAI, di cui non posso certo parlarvi io qui nella sua capitale. Da che mondo è mondo - si è detto e ridetto in questi anni - la cultura non produce ricchezza; anche i maggiori musei, dal Moma di New York ai Musei Vaticani, da soli non ce la fanno. D'altra parte (questo lo sappiamo) il patrimonio culturale non si tutela per produrre ricchezza, ma per un fine più alto, che è quello della crescita culturale dei cittadini; quindi, il patrimonio può essere gestito in perdita e questa perdita deve essere socialmente accettata (o dovremmo dire accettabile).

Penso anche però che non da oggi sia chiaro che l'economia legata ai beni culturali risiede nella ricchezza prodotta in termini 'immateriali' e di più lungo respiro: dobbiamo calcolare infatti la minore spesa generata nel medio e lungo periodo da quella che possiamo definire come una 'tutela sociale attiva', cioè da una crescita culturale e partecipativa della

23 M. Montella, Valorizzare per tutelare, in G. Volpe (a cura di), Patrimoni culturali e paesaggi di Puglia e d'Italia tra conservazione e innovazione, Atti delle Giornate di studio (Foggia 30 settembre e 22 novembre 2013), Bari 2014, 83. 
società nel suo complesso, che porta a comportamenti responsabili, rispettosi dei monumenti, del paesaggio e dell'ambiente, che nel calcolo economico generale producono vantaggi consistenti e duraturi.

Quando si parla di redditività dei beni culturali sappiamo quindi che questo reddito non deriva tanto dal loro diretto sfruttamento, per esempio attraverso i biglietti di ingresso o i diritti sulle riproduzioni, che producono peraltro introiti irrilevanti. Il conto va fatto in ragione dell'indotto generato dalle attività che ruotano attorno al patrimonio, in termini di crescita dell'occupazione e del fatturato nei servizi e nel commercio connessi al turismo di qualità. È un reddito quindi che alimenta le attività dei singoli ed anche le finanze pubbliche attraverso la fiscalità generale $e^{24}$.

Ma l'esperienza del FAI ci insegna che la gestione diretta dei beni può produrre ricchezza pulita e garantire anche gestioni sostenibili, come dimostra anche l'esperienza degli anni migliori dei Parchi della Val di Cornia in Toscana, che ho conosciuto più da vicino, così come espressa nel loro Bilancio sociale ${ }^{25}$. Esistono infatti casi virtuosi, che si manifestano spesso tanto meglio quanto più in autonomia rispetto alla gestione pubblica del patrimonio culturale. Questa non è certo una constatazione consolante, ma indica che c'è spazio per una innovazione che ci affranchi dalla morsa di una scelta che vorrebbe sottrarre i beni culturali alle leggi dell'economia per sacralizzarli sull'altare di una cultura esente da commerci, e una pratica dell'amministrazione pubblica di questo patrimonio spesso indolente, gelosa, inefficiente.

Le ricerche archeologiche condotte a Populonia, l'antica città etrusca sulla costa toscana, hanno portato all'apertura del nuovo Parco dell'Acropoli, visitato da decine di migliaia di turisti. La Val di Cornia ha avuto, nel secolo scorso, un grande sviluppo industriale, che vedeva nella città-fabbrica l'unica possibilità di crescita. Negli anni ' 80 la crisi dell'acciaio rese urgente una ristrutturazione dell'economia del territorio, diversificando le attività e incentivando la piccola imprenditorialità. La ricchezza dei beni culturali ed ambientali fu vista allora come una risorsa strategica per la riconversione e lo sviluppo di un turismo basato sulla valorizzazione del territorio. È sorto così il Sistema dei Parchi

24 Manacorda, L'Italia, 82-83.

25 Tra Cultura e Ambiente: verso un Bilancio sociale per la Parchi Val di Cornia SpA, Milano, Il Sole 24 Ore, 2009. 
della Val di Cornia, che comprende 6 aree di grande pregio ed un museo nella città di Piombino ${ }^{26}$.

Queste aree sono gestite dalla Parchi Val di Cornia, una società a capitale pubblico, che ha il compito di progettare gli interventi di valorizzazione e i servizi per l'accoglienza (centri visita, didattica, percorsi naturalistici ed archeologici, bookshop, centri ristoro, ostelli...), per i quali sono stati spesi negli anni oltre 25 milioni di euro, coperti da contributi nazionali ed europei, da capitale pubblico locale e in parte anche da capitali privati. Pochi anni dopo l'inaugurazione del primo lotto del parco, è stata aperta al pubblico anche l'acropoli di Populonia, grazie ad una collaborazione fra il Mibact, gli Enti locali e le Università, che hanno lavorato insieme con la Società dei Parchi, ciascuno secondo le proprie competenze e responsabilità: insomma, un caso significativo di integrazione tra ricerca scientifica, pianificazione urbanistica e politiche ambientali e culturali.

Tra i punti di forza del Sistema dei Parchi è l'offerta turistica poliedrica, che integra le risorse storico-culturali, ambientali e paesaggistiche con l'attività balneare e di sport e svago nella natura, per non parlare di una offerta enogastronomica di alta qualità. Questa integrazione, la gestione d'impresa, le economie di scala hanno determinato una costante crescita della capacità di autofinanziamento della Società, con un rapporto ricavi/costi che nel 2007 ha sfiorato il pareggio di bilancio. Gli indicatori turistici mostrano come la Val di Cornia si sia collocata al primo posto nella Regione Toscana per il tasso di sviluppo del turismo ${ }^{27}$.

In questi ultimi anni i segnali di una crisi, che va inserita nelle difficoltà finanziarie globali ma che ha anche ragioni localistiche, si manifestano attraverso un inquietante rallentamento della ricerca, la riduzione delle manutenzioni e dei restauri, la sospensione degli investimenti in valorizzazione e comunicazione. Occorre invece proprio il contrario, e cioè consolidare il quadro degli interventi, garantire la continuità del

26 D. Manacorda, Archeologia e architettura per il Parco archeologico di Populonia, in AA.VV. (a cura di), arch.it.arch. Dialoghi di archeologia e architettura 2005-2006, Roma 2009, 136-153.

27 D. Manacorda, Patrimonio bistórico, turismo, economía: ¿un desafío o una alianza? El caso de Populonia (Toscana, Italia), in I. Arrieta Urtizberea (a cura di), Museos y turismo: expectativas y realidades, Bilbao 2012, 85-100. 
recupero, assicurarsi le risorse necessarie a mantenere in efficienza quanto sinora messo in piedi ${ }^{28}$.

I Parchi della Val di Cornia sono stati un vero e proprio modello per l'innovazione in questo settore ${ }^{29}$. Qualcuno potrebbe osservare che riempirsi la bocca con la parola innovazione è facile, certo. Ma che vogliamo dire? Per esempio che è giunta l'ora di fare alcune scelte culturali di fondo nella amministrazione del nostro patrimonio di storia, arte, archeologia ${ }^{30}$. Quali? Dobbiamo optare per:

- una tutela contestuale, che non separi i beni da tutelare in base alle discipline (arte, archeologia, architettura), ma li consideri unitariamente nel loro contenitore che è il paesaggio storico; è il traguardo che il ministro Franceschini ha avuto il grande merito di mettere a fuoco e attuare coraggiosamente (con tutte le difficoltà che conosciamo);

- una tutela come sistema inclusivo e servizio pubblico, che non può limitarsi ad impedire ai cittadini di essere cattivi, ma deve metterli in condizione di essere buoni, di usare il loro patrimonio (chiunque abiti in una casa sa che il modo migliore per mantenerla è viverci: il degrado comincia il giorno dopo che ci siamo chiusi la porta alle nostre spalle);

- una tutela come luogo della ricerca e della formazione, costringendo Mibact e Miur a stringere accordi per una formazione condivisa (si parla di una cosa bellissima, i Policlinici dei beni culturali ${ }^{31}$, che ha il merito di aver tradotto in programma politico tanti aspetti del dibattito attuale), anche se su questo tema specifico credo che purtroppo stiamo registrando un passo avanti e tre indietro;

- una tutela come democratizzazione della cultura, come addio a una concezione gelosa del patrimonio: democrazia non è proprietà pubblica del patrimonio, magari reso pubblicamente inaccessibile, ma è

28 D. Manacorda, Una stagione di ricerche a Populonia e la sua crisi, in V. Di Cola e F. Pitzalis (a cura di), Materiali per Populonia 11, Pisa 2015, 5-16.

29 M. Cerquetti, L'innovazione del prodotto culturale in chiave multidimensionale e multistakeholder: il caso del Sistema Parchi val di Cornia, Il Capitale culturale, 4 (2012), 31-68.

30 D. Manacorda, L'innovazione nei beni culturali, in G. Volpe (a cura di), Patrimoni culturali, 75-81; Id., Archeologia globale e sistema della tutela, Archeologia Medievale, 41 (2014), 141-148.

31 Si veda comunque in proposito Volpe, Patrimonio, 44-46. 
cultura pubblica, coltivazione pubblica del patrimonio attraverso i cittadini stessi;

- tutela come massima circolazione dei dati, come diffusione di informazioni, come libero accesso ai beni (compresi quelli bibliografici e archivistici, sinora assurdamente esclusi dalla liberalizzazione introdotta dall'Art bonus ${ }^{32}$ ), perché producano a loro volta valore e cultura;

- insomma tutela come chiamata a raccolta di tutte le energie positive del paese.

In questo senso è evidente il ruolo che potrebbe avere l'associazionismo culturale, uno degli strumenti più sani di cui l'Italia può dotarsi per alleviare l'affanno dell'intervento pubblico sul patrimonio. Ed è ancor più evidente il ruolo che può avere l'impresa, se la gestione del patrimonio potesse cominciare ad essere sempre più affidata - nei mille casi in cui la mano pubblica non ce la fa - alle migliori energie imprenditoriali, con e senza fine di lucro, e al volontariato. Ma senza mettere in contrapposizione questa bellissima esperienza civile con i diritti dei giovani, che dopo anni di formazione chiedono di veder riconosciute le proprie competenze in un campo dove di spazio ce ne sarebbe davvero per tutti ${ }^{33}$.

E quindi penso che non possiamo relegare nel mondo delle chimere la possibilità che l'amministrazione pubblica torni ad aprire le porte ai giovani perché portino aria fresca nelle mentalità prima ancora che nelle procedure. Siamo quindi felici che un po' di energie vengano introdotte dal prossimo concorso per 500 posti che tapperanno alcune delle falle del sistema. Ma se non dobbiamo stancarci di chiedere più personale per l'amministrazione, e più personale tecnico (perché non servono uffici abitati solo da qualche funzionario e da uno stuolo di custodi non qualificati) dobbiamo dire, oggi più che mai, che tra il

32 Il movimento "Fotografie libere per i Beni Culturali" (https://fotoliberebbcc.wordpress.com/) ha raccolto migliaia di firme di studiosi e funzionari della pubblica amministrazione a sostegno della libera riproduzione a distanza con mezzi digitali propri di beni bibliografici e archivistici, nel rispetto delle norme a tutela della privacy, del diritto di autore e dell'integrità del bene. Si veda in proposito: M. Modolo, Il sogno infranto delle libere riproduzioni, Il Giornale dell'Arte, settembre 2014, 10; M. Modolo - A. Tumicelli, Una possibile riforma sulla riproduzione dei beni bibliografici ed archivistici, Aedon, 1 (2016).

33 Manacorda, L'Italia, 50-51. 
posto al ministero e il lavoro sottopagato camuffato da volontariato (che è cosa ben diversa dal volontariato vero, che è invece una benedizione, perché lega i cittadini alle sorti del loro patrimonio), tra questi due mondi ci sono di mezzo le praterie della libera iniziativa individuale e associativa. Là dove la mano pubblica non ce la fa ad essere presente con l'efficacia e la dignità richieste, si aprano le porte della gestione di siti, monumenti e musei a chi ha la creatività e il coraggio di assumersi questo compito di ricucitura del patrimonio con la società, di 'rammendo' come dice Renzo Piano ${ }^{34}$, e ben venga se questo produce, con la diffusione della cultura, anche una ricchezza pulita. Se finalmente qualcosa si muove per recuperare il patrimonio edilizio delle nostre belle case cantoniere, si potrà pur fare qualcosa per fermare l'abbandono di una infinità di siti e monumenti, riportati alla vita in forme compatibili con la loro natura? E questo - sulla scia della Convenzione di Faro ${ }^{35}$ - promuovendo principi per la gestione sostenibile e la manutenzione del patrimonio, ponendo l'accento su questo, come ci insegnava trenta anni fa, inascoltato, Giovanni Urbani ${ }^{36}$ e non - sacralmente - sulla conservazione e il restauro, concetti nobilissimi, certo, ma che sono risultati spesso alternativi, se non antitetici, ai primi.

Insomma, più personale e risorse nell'Amministrazione pubblica, massima apertura al volontariato vero, porte spalancate ad attività di impresa incoraggiate ed assistite non sono in contrasto tra di loro, sono il tridente che potrebbe farci superare le casematte opposte da una concezione feticistica del patrimonio, paurosa del presente e terrorizzata dal futuro, di cui siamo stati a lungo ostaggi nell'amministrazione, nella politica e anche in molti mezzi di comunicazione di massa.

La concezione sacrale del patrimonio è il collante ideologico di un conservatorismo che esprime angosce comprensibili e propone scelte politiche spesso di semplice buon senso, ma pur sempre di conservatorismo si tratta, perché, prodotta una diagnosi del male, sorvola sull'anamnesi, cioè sulla ricerca dei motivi che lo hanno generato. E quando la fa, li trova nei fattori esterni all'organismo che si dovrebbe curare,

34 R. Piano, Il rammendo delle periferie, Il Sole 24 ore, 26 gennaio 2014.

35 Si vedano in proposito gli Atti del Convegno su La valorizzazione dell'eredità culturale in Italia (Macerata 5 e 6 novembre 2015), Il Capitale culturale, Suppl. 5 (2016), a cura di P. Feliciati.

36 G. Urbani, Intorno al restauro, a cura di B. Zanardi, Milano 2000. 
non nei deficit interni, nelle categorie culturali, nella mentalità, nell'organizzazione delle strutture che hanno in carico il nostro patrimonio culturale, e nelle relative procedure. Questo conservatorismo confonde non solo il senso profondo del tanto sbandierato articolo 9 della nostra Costituzione, ma dimentica l'art. 33, che sancisce la libertà della ricerca, per non parlare dell'articolo 118, che chiede a Stato, Regioni, Province, e Comuni di favorire, sulla base del principio di sussidiarietà, "l'autonoma iniziativa dei cittadini, singoli e associati, per lo svolgimento di attività di interesse generale". Lettera morta per l'amministrazione pubblica dei beni culturali.

Questi tre articoli sono un combinato disposto che io leggo così: che il compito della amministrazione pubblica dovrebbe essere quello di garantire la tutela, favorire la ricerca da parte di tutti e orientare la valorizzazione, dando aria alla creatività degli italiani. E' questa la concezione aperta e pluralistica che, accanto alle istituzioni della democrazia rappresentativa, dà spazio ad associazioni e fondazioni che organizzino una partecipazione dei cittadini su ambiti definiti della vita pubblica, ponendosi come corpi intermedi fra cittadinanza e governi ${ }^{37}$.

Se posso usare uno slogan, direi che occorre passare dalla resistenza alla liberazione, che metta in discussione un passato idealizzato, e dia vita ad un pacchetto organico di idee innovative, attorno al quale raccogliere quanti operano generosamente nel settore e il mare della società civile, con tutte le sue sfaccettature, anche quelle che sembrano stare dalla parte opposta del poliedro in cui operiamo.

Ma dobbiamo cercare di capire le motivazioni profonde delle diverse opzioni in campo. Nel Mibact (e non solo) convivono infatti due anime: una che punta, con qualche strappo, ad una riforma inevitabile e da tempo attesa, l'altra che - ad esempio con le circolari che sono partite come missili dalla Direzione Generale Archeologia ${ }^{38}$ - sem-

37 A. Carandini, Il FAI per la Puglia e per l'Italia: il ruolo dell'associazionismo e della partecipazione dei cittadini, in G. Volpe (a cura di), Patrimoni culturali, 159-167.

38 Si vedano a titolo di esempio le circolari della Direzione Generale Archeologia n. 03/2015 del 9.2.2015 e n. 06/2016 del 15.2.2016 a proposito delle concessioni di scavo; sul tema si veda G. Volpe, A proposito delle concessioni di scavo e dei rapporti tra Università e Soprintendenze, Post Classical Archaeologies, 3 (2013), 301 310; D. Manacorda, Concessioni di scavo, in M. Montella (a cura di), Economia e gestione, 164-166. 
brano volerci ricordare quanto sia abissale il distacco di quelle stanze dalla realtà del Paese. E' in atto un confronto culturale e politico che attraversa gli abituali schieramenti. La burocrazia ministeriale più chiusa trova negli anatemi di certi maîtres à penser un appoggio insperato. La gran massa degli addetti ai lavori chiede, in modo diverso ma convergente, di essere messa in condizioni di lavorare per il meglio per il bene comune. Sta alla politica consentirgli di farlo. Sol che si abbia il coraggio di dirsi che le politiche per i beni culturali non possono essere politiche settoriali, perché hanno in sé una visione complessiva che abbraccia la politica economica nel suo insieme, quella ambientale, delle infrastrutture, dei servizi sociali, della formazione.

Non dubito che il ministro Franceschini questa consapevolezza la abbia; così come mi sembra chiaro che il coro delle proteste che ha accompagnato le sue mosse questa consapevolezza spesso non la abbia. E tuttavia, quando le voci allarmate sono dettate da esperienza e spirito di servizio, dobbiamo saperle ascoltare, non per frenare ma per andare avanti, non per guardarsi come rivali dalle due diverse sponde, ma per attraversare il guado in massa, andando anche quel po' controcorrente quanto è necessario per raggiungere la riva. 